\title{
Dark matter and muon $(g-2)$ in an extended Ma model
}

\section{Seungwon Baek*}

School of Physics, KIAS, 85 Hoegiro, Seoul 02455, Korea

E-mail: swbaekakias.re.kr

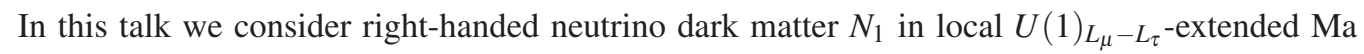
model. With the light $U(1)_{\mu-\tau}$ gauge boson $\left(m_{Z^{\prime}} \sim \mathscr{O}(100) \mathrm{MeV}\right)$ and small $U(1)_{\mu-\tau}$ gauge coupling $\left(g_{Z^{\prime}} \sim 10^{-4}-10^{-3}\right)$ which can accommodate the muon $(g-2)$ anomaly and is still allowed by other experimental constraints, we show that we can get correct relic density of dark matter for wide range of dark matter mass $\left(M_{1} \sim 10-100 \mathrm{GeV}\right)$, although the gauge coupling constant $g_{Z^{\prime}}$ is small. This is due to the fact that the annihilation cross section of dark matter pair is enhanced by $M_{1}^{4} / m_{Z^{\prime}}^{4}$ in the processes $N_{1} N_{1} \rightarrow Z^{\prime} Z^{\prime}$ or $N_{1} N_{1} \rightarrow Z^{\prime} H_{2}$. We also consider the constraints from direct detection, collider searches.

The 11th International Workshop Dark Side of the Universe 2015

14-18 December 2015

Kyoto, Japan

\footnotetext{
${ }^{*}$ Speaker.
} 


\begin{tabular}{||c||c|c|c|c|c|c|c|c|c||c|c|c||}
\hline \hline & $L_{e}$ & $L_{\mu}$ & $L_{\tau}$ & $e_{R}^{c}$ & $\mu_{R}^{c}$ & $\tau_{R}^{c}$ & $N_{e}^{c}$ & $N_{\mu}^{c}$ & $N_{\tau}^{c}$ & $\Phi$ & $\eta$ & $S$ \\
\hline \hline$S U(2)_{L}$ & \multicolumn{3}{||}{2} & \multicolumn{3}{|c|}{1} & \multicolumn{3}{|c||}{1} & 2 & 2 & 1 \\
\hline$U(1)_{Y}$ & \multicolumn{3}{|c|}{$-1 / 2$} & \multicolumn{3}{|c|}{1} & \multicolumn{3}{c||}{0} & $+1 / 2$ & $+1 / 2$ & 0 \\
\hline$U(1)_{L_{\mu}-L_{\tau}}$ & 0 & +1 & -1 & 0 & -1 & +1 & 0 & -1 & +1 & 0 & 0 & +1 \\
\hline$Z_{2}$ & \multicolumn{3}{|c|}{+} & \multicolumn{3}{|c|}{+} & \multicolumn{3}{|c|}{-} & + & - & + \\
\hline \hline
\end{tabular}

Table 1: The particle content and the charge assignment under $S U(2)_{L} \times U(1)_{Y} \times U(1)_{L_{\mu}-L_{\tau}} \times Z_{2}$.

\section{Introduction}

About 27\% of the universe is composed of dark matter, but we do not know its nature yet. We may, however, find a clue for the dark matter in other sector of the standard model (SM), such as neutrino sector. One example is the models where the neutrino masses are generated radiatively with dark matter as an essential component [1].

In Ref. [2], we extended Ma's scotogenic model [3] so that the model has gauged $L_{\mu}-L_{\tau}$ symmetry. In fact, three symmetries $L_{e}-L_{\mu}, L_{e}-L_{\tau}$, and $L_{\mu}-L_{\tau}$, where $L_{i}$ is the lepton number associated with the flavor $i$, can be gauged without the extension of the SM particle content ${ }^{1}$. The gauge anomaly cancels between different generations. In that paper we demonstrated that the neutrino mass matrix has two-zero texture due to the gauge symmetry, making the theory very predictive. Especially we predicted the neutrino masses have inverted hierarchy and the Dirac CP phase is close to maximal $\left(\sim 270^{\circ}\right)$.

In this talk we consider the dark matter phenomenology of the model [4]. Especially we will show that we can get correct dark matter relic abundance and explain the muon $(g-2)\left((g-2)_{\mu}\right)$ anomaly at the same time. According to [5], almost all the region which can explain $(g-2)_{\mu}$ is excluded by the neutrino trident production in $U(1)_{\mu-\tau}$ model. However, the region for $Z^{\prime}$ mass, $m_{Z^{\prime}} \lesssim 400 \mathrm{MeV}$, and for the extra $U(1)$ gauge coupling, $g_{Z^{\prime}} \sim 3 \times 10^{-4}-10^{-3}$, is still allowed and can accommodate $(g-2)_{\mu}$ anomaly. In this talk we concentrate on this region, since the current experimental results still show 3-4 $\sigma$ deviation from the SM predictions.

The analysis is applicable to more general dark matter models with light $Z^{\prime}$ gauge boson coupled to right-handed neutrinos where the lightest right-handed neutrino is the dark matter candidate. For example, the inert doublet scalar in the Ma model is irrelevant for our discussion on dark matter and we would get similar results with this talk if only the right-handed neutrinos have similar structure.

\section{The model}

The original Ma model [3] introduces right-handed neutrinos $N_{i}^{c}(i=e, \mu, \tau)$, and $S U(2)_{L^{-}}$ doublet scalar $\eta$, both of which are odd under discrete symmetry $Z_{2}$. As a consequence the lightest

\footnotetext{
${ }^{1}$ We will denote $L_{\mu}-L_{\tau}$ as just $\mu-\tau$ for notational simplicity.
} 
state of them do not decay into the standard model (SM) particles and can be a dark matter candidate. The Yukawa interactions involving $L, N^{c}, \eta$ fields in the original Ma models are given by

$$
\mathscr{L}=-\frac{1}{2} M_{i j} N_{i}^{c} N_{j}^{c}-y_{i j} \Phi^{\dagger} L_{i} e_{j}^{c}+f_{i j} \eta \cdot L_{i} N_{j}^{c},
$$

where $\Phi$ is the SM Higgs doublet field and $\eta \cdot L_{i} \equiv \varepsilon^{a b} \eta_{a} L_{i b}$ in $S U(2)_{L}$ space. The neutrino mass terms come from one one-loop diagrams involving both $N_{i}^{c}$ and $\eta$ [3].

To extend the Ma model to local $U(1)_{\mu-\tau}$ symmetry, we just need to introduce one additional scalar particle $S$ charged under $U(1)_{\mu-\tau}$ to break the abelian symmetry spontaneously. The particle content and the charge assignment under $S U(2)_{L} \times U(1)_{Y} \times U(1)_{\mu-\tau} \times Z_{2}$ are shown in Table 1 .

The new gauge interactions are dictated by the gauge covariant derivative to give

$$
\Delta \mathscr{L}=\sum_{\psi=l_{L}^{f}, e_{R}^{f}, N_{R}^{f}} g_{Z^{\prime}} Q_{\psi}^{\prime} \bar{\psi} \gamma^{\mu} Z_{\mu}^{\prime} \psi
$$

where $f=\mu, \tau$.

Due to $U(1)_{\mu-\tau}$ symmetry all the terms in (2.1) are not allowed. And the Yukawa interaction and right-handed neutrino mass terms become more restricted to be

$$
\begin{aligned}
\mathscr{L} & =-\frac{1}{2} M_{e e} N_{e}^{c} N_{e}^{c}-\frac{1}{2} M_{\mu \tau}\left(N_{\mu}^{c} N_{\tau}^{c}+N_{\tau}^{c} N_{\mu}^{c}\right) \\
& -h_{e \mu}\left(N_{e}^{c} N_{\mu}^{c}+N_{\mu}^{c} N_{e}^{c}\right) S-h_{e \tau}\left(N_{e}^{c} N_{\tau}^{c}+N_{\tau}^{c} N_{e}^{c}\right) S^{*} \\
& +\eta \cdot\left(f_{e} L_{e} N_{e}^{c}+f_{\mu} L_{\mu} N_{\mu}^{c}+f_{\tau} L_{\tau} N_{\tau}^{c}\right) \\
& -\Phi^{\dagger}\left(y_{e} L_{e} e_{R}^{c}+y_{\mu} L_{\mu} \mu_{R}^{c}+y_{\tau} L_{\tau} \tau_{R}^{c}\right) \\
& + \text { h.c },
\end{aligned}
$$

where all the fermions are Weyl spinors. After $S$ gets vev $v_{S}\left(\langle S\rangle=v_{S} / \sqrt{2}\right)$, we can see that the mass matrix of the right-handed neutrinos can be written as

$$
M_{R}=\left(\begin{array}{ccc}
M_{e e} & \frac{1}{2} h_{e \mu} v_{S} & \frac{1}{2} h_{e \tau} v_{S} \\
\frac{1}{2} h_{e \mu} v_{S} & 0 & M_{\mu \tau} e^{i \theta_{R}} \\
\frac{1}{2} h_{e \tau} v_{S} & M_{\mu \tau} e^{i \theta_{R}} & 0
\end{array}\right)
$$

By appropriate phase rotation, we can make all the parameters real except the one in $(2,3)$ component for which we allow CP violating phase $\theta_{R}$. The matrix $M_{R}$ is symmetric and can be diagonalized by a unitary matrix

$$
V^{T} M_{R} V=\operatorname{diag}\left(M_{1}, M_{2}, M_{3}\right) .
$$

The scalar potential of $\Phi, \eta$, and $S$ is given by

$$
\begin{aligned}
V & =\mu_{\Phi}^{2}|\Phi|^{2}+\mu_{\eta}^{2}|\eta|^{2}+\mu_{S}^{2}|S|^{2} \\
& +\frac{1}{2} \lambda_{1}|\Phi|^{4}+\frac{1}{2} \lambda_{2}|\eta|^{4}+\lambda_{3}|\Phi|^{2}|\eta|^{2}+\lambda_{4}\left|\Phi^{\dagger} \eta\right|^{4}+\frac{1}{2} \lambda_{5}\left[\left(\Phi^{\dagger} \eta\right)^{2}+\text { h.c. }\right] \\
& +\frac{1}{2} \lambda_{6}|S|^{4}+\lambda_{7}|\Phi|^{2}|S|^{2}+\lambda_{8}|\eta|^{2}|S|^{2} .
\end{aligned}
$$


After $\Phi$ and $S$ get vev, $v$ and $v_{S}$, respectively, we can write

$$
\Phi=\left(\begin{array}{c}
0 \\
\frac{1}{\sqrt{2}}(v+h)
\end{array}\right), \quad S=\frac{1}{\sqrt{2}}\left(v_{S}+s\right),
$$

in the unitary gauge. Then the two neutral states $h$ and $s$ can mix with each other with mixing angle $\alpha$, whose mass eigenstates we denote as $H_{1}$ and $H_{2}$ with masses $m_{H_{1}}$ and $m_{H_{2}}$, respectively [6]. Here $H_{1}$ is the SM-like Higgs boson with $m_{H_{1}} \approx 125 \mathrm{GeV}$. We will assume this "Higgs portal" term, i.e. the $\lambda_{7}$, is small, because its mixing angle is strongly suppressed by the study of Higgs signal strength [6].

\section{Muon $(g-2)$, relic density, direct detection of dark matter, and other tests of the model}

In this section we concentrate on the dark matter phenomenology, especially the relic density and the direct detection, of the model in the region which can explain the muon $(g-2)$ anomaly. Let us first consider the muon $(g-2)$ in our model. The discrepancy between experimental measurement [7] and the SM prediction [8]

$$
\Delta a_{\mu} \equiv a_{\mu}^{\exp }-a_{\mu}^{\mathrm{SM}}=(295 \pm 88) \times 10^{-11},
$$

is about $3.4 \sigma$ and can be explained by the $U(1)_{\mu-\tau}$ gauge boson contribution $[9,10]$. Although the neutrino trident production process disfavors the $Z^{\prime}$ explanation of muon $(g-2)$ for $m_{Z^{\prime}} \gtrsim 0.4$ $\mathrm{GeV}$ [5], the light $Z^{\prime}$ region is still consistent with $(g-2)_{\mu}$.

According to the Ref. [5], the allowed region for $(g-2)_{\mu}$ is characterized by light $Z^{\prime}, m_{Z^{\prime}} \lesssim 0.4$ $\mathrm{GeV}$ and small $Z^{\prime}$ gauge coupling constant, $10^{-4} \lesssim g_{Z^{\prime}} \lesssim 10^{-3}$. For this small gauge coupling constant, it is naively expected the annihilation processes of the dark matter pair at the electroweak scale dominated by [11]

$$
\begin{aligned}
& N_{1} N_{1} \rightarrow Z^{\prime *} \rightarrow l^{+} l^{-}, v_{l} \bar{v}_{l} \quad(l=\mu, \tau), \\
& N_{1} N_{1} \rightarrow Z^{\prime} Z^{\prime},
\end{aligned}
$$

would have very small cross sections. As a consequence, the dark matter relic density would overclose the universe. It turns out that this is not the case.

The dominant dark matter annihilation processes in our region of interest (i.e. light $Z^{\prime}$ and small $\left.g_{Z^{\prime}}\right)$ are

$$
N_{1} N_{1} \rightarrow Z^{\prime} Z^{\prime}, \quad \text { and } \quad \mathrm{N}_{1} \mathrm{~N}_{1} \rightarrow Z^{\prime} \mathrm{H}_{2}
$$

where $H_{2}$ is the lighter mass eigenstate between the SM Higgs and the $U(1)_{\mu-\tau}$ breaking scalar. For the second process to occur, $\mathrm{H}_{2}$ should also be light enough to be kinematically allowed. The relevant diagrams are shown in Fig. 1.

We notice that the longitudinal $Z^{\prime}$ polarization has enhancement factor, $\varepsilon^{* \mu}(p) \sim p^{\mu} / m_{Z^{\prime}}$, when its energy is much larger than its mass. Since the total energy scale is almost fixed by the dark matter mass in dark matter annihilation, there is an enhancement factor $M_{1} / m_{Z^{\prime}}$ for each $Z^{\prime}$ 


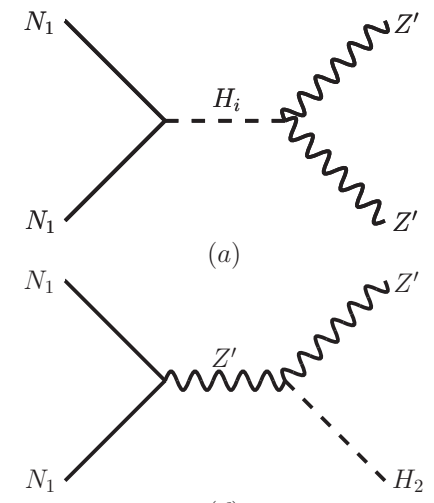

$(d)$

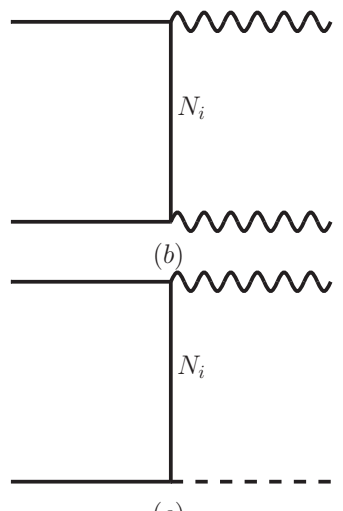

$(e)$
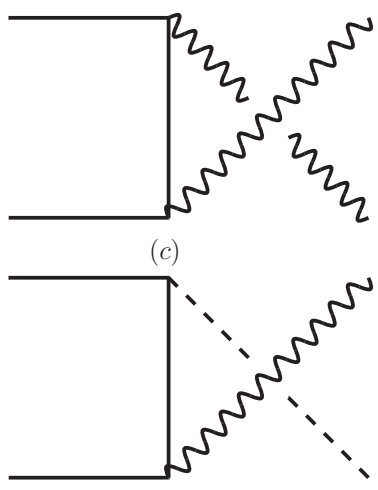

$(f)$

Figure 1: Feynman diagrams for the processes, $N_{1} N_{1} \rightarrow Z^{\prime} Z^{\prime}$ and $N_{1} N_{1} \rightarrow Z^{\prime} H_{2}$. Here $H_{i}(i=1,2)$ are two scalar mass eigenstates and $N_{i}(i=1,2,3)$ are three right-handed neutrino mass eigenstates.

in the external or internal line in the annihilation diagram. Consequently the diagrams with two $Z^{\prime}$ gauge boson lines are most enhanced. And the enhancement factor in the annihilation cross section is $M_{1}^{4} / m_{Z^{\prime}}^{4}$. This large enhancement can compensate the suppression due to small gauge coupling constant $g_{Z^{\prime}}$ allowed by the $(g-2)_{\mu}$.

We scanned the region which can explain muon $(g-2)$ anomaly in $\left(m_{Z^{\prime}}, g_{Z^{\prime}}\right)$ plane [5], which can also be seen in the right panel of Fig. 3. For other parameters, we set

$$
\begin{aligned}
\alpha & =10^{-7}, \\
m_{H_{1}} & =125 \mathrm{GeV}, \\
\lambda_{2} & =\lambda_{3}=\lambda_{8}=1, \\
m_{\eta^{ \pm}} & =m_{\eta_{R}}=m_{\eta_{I}}=10 \mathrm{TeV},
\end{aligned}
$$

where $m_{\eta^{ \pm}}$and $m_{\eta_{R}(I)}$ are charged- and neutral-masses from inert scalar doublet $\eta^{2}$. The change of the above parameters does not change our results much. And we scanned in the range

$$
\begin{aligned}
& 0<m_{H_{2}} \quad<\sqrt{4 \pi} m_{Z^{\prime}} / g_{Z^{\prime}} \\
& 10 \mathrm{GeV}<M_{e e}, M_{\mu \tau}<100 \mathrm{GeV} \\
& -4 \pi<h_{e \mu}, h_{e \tau}<4 \pi \\
& -\pi<\theta_{R}<\pi,
\end{aligned}
$$

where used $m_{H_{2}} \approx \sqrt{\lambda_{6}} v_{S}$ to set the maximum value of $m_{H_{2}}$. With this scan, we get $M_{1} \lesssim 100 \mathrm{GeV}$ and $M_{2} \lesssim 3000 \mathrm{GeV}$ as we can see in Fig. 2 .

Fig. 2 shows the relic density versus $M_{1}$ (left panel) and $M_{2}$ (right panel). The horizontal lines represent $\pm 5 \sigma$ values of Planck result, $\Omega h^{2}=0.1199 \pm 0.0027$ [12]. We can see that the current relic density can be explained for wide range of dark matter mass, $M_{1} \gtrsim 5 \mathrm{GeV}$ (See also the left figure in Fig. 3). We can also see that the $t$-channel $N_{2}$ contribution which is not suppressed by $v_{\text {rel }}^{2}$ can be important if it is not too heavy.

\footnotetext{
${ }^{2}$ The neutrino masses are sensitive to Yukawa couplings $f_{i}(i=e, \mu, \tau)$ in (2.3) and are not strongly correlated with the dark matter phenomenology
} 

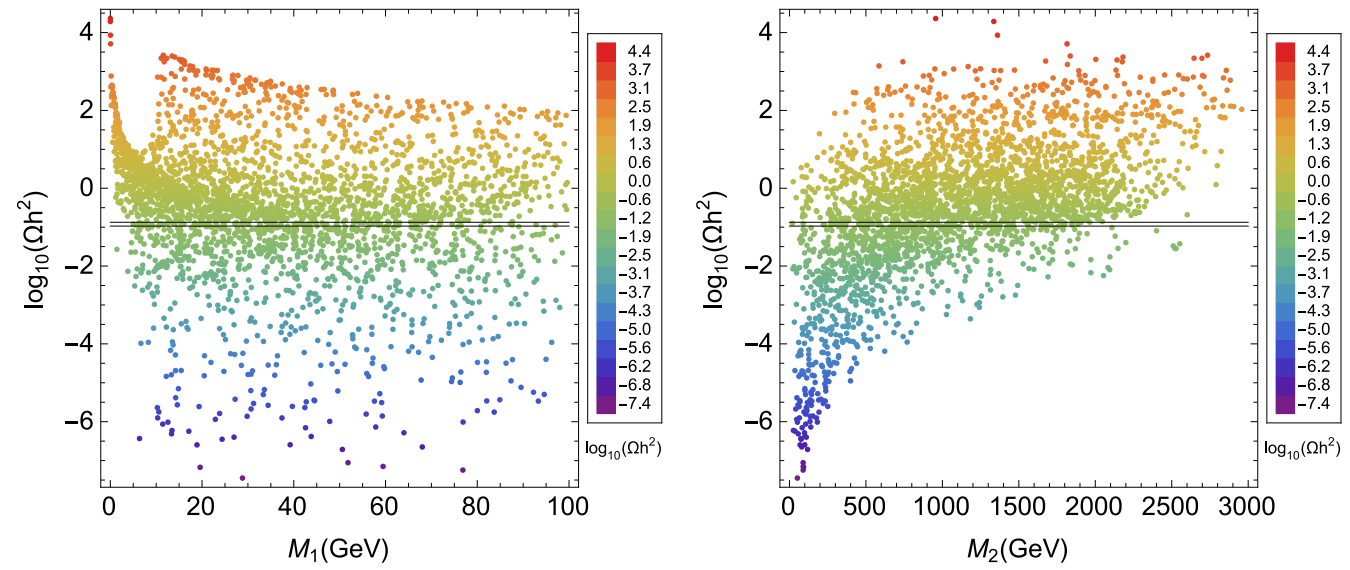

Figure 2: The relic density versus $M_{1}$ (left panel) and $M_{2}$ (right panel). The horizontal lines represent $\pm 5 \sigma$ values of Planck result, $\Omega h^{2}=0.1199 \pm 0.0027$.
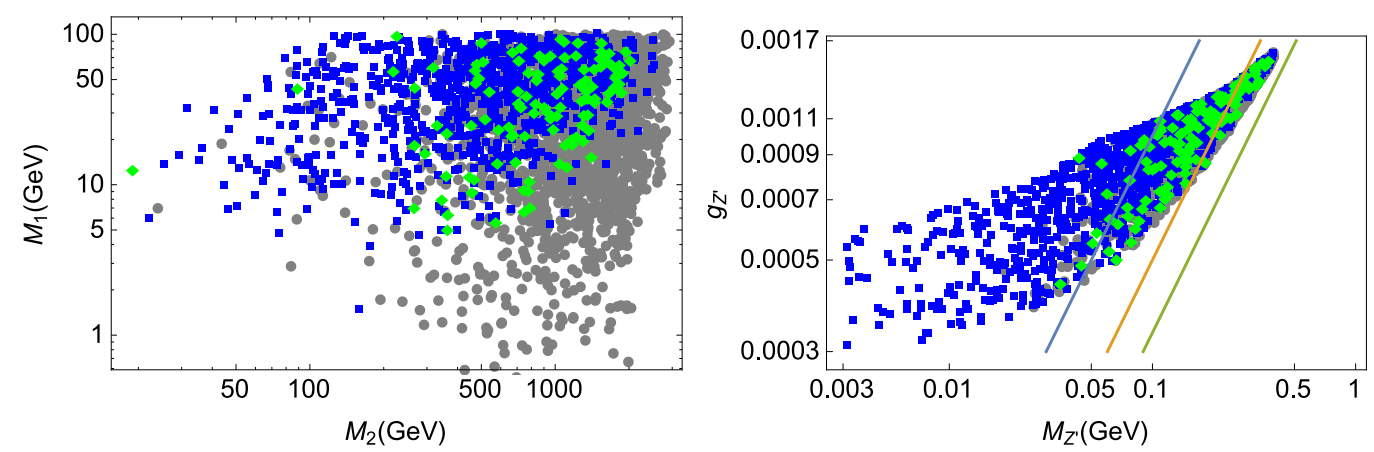

Figure 3: Scatter plots in $\left(M_{2}, M_{1}\right)$ plane (left panel) and $\left(m_{Z^{\prime}}, g_{Z^{\prime}}\right)$ plane (right panel). All the points can explain the $(g-2)_{\mu}$ at $2 \sigma$ level. The green points satisfy $0.1<\Omega h^{2}<0.14$, the blue points $\Omega h^{2}<0.1$, and the gray points $\Omega h^{2}>0.14$. In the right panel the straight lines correspond to $m_{Z^{\prime}} / g_{Z^{\prime}}=100,200,300 \mathrm{GeV}$ from the left.

In Fig. 3, we show scatter plots in $\left(M_{2}, M_{1}\right)$ plane (left panel) and $\left(m_{Z^{\prime}}, g_{Z^{\prime}}\right)$ plane (right panel). All the points can explain the $(g-2)_{\mu}$ at $2 \sigma$ level. The green points satisfy $0.1<\Omega h^{2}<0.14$, the blue points $\Omega h^{2}<0.1$, and the Gray points $\Omega h^{2}>0.14$. In the right panel the straight lines correspond to $M_{Z^{\prime}} / g_{Z^{\prime}}=100,200,300 \mathrm{GeV}$ from the left. We can see that the relic abundance of our universe can be explained if $N_{1}$ is not too light (i.e. if $M_{1} \gtrsim 5 \mathrm{GeV}$ ) and $N_{2}$ has electroweak scale mass. The right panel shows that the correct relic density can be obtained if $Z^{\prime}$ is not too light. If $Z^{\prime}$ is too light, i.e. $m_{Z^{\prime}} \lesssim 40 \mathrm{MeV}$, the annihilation cross section becomes too large and the relic density becomes too small.

Since $Z^{\prime}$ does not couple to quarks directly, our model does not have tree-level diagram for the direct detection of dark matter off nucleons. At one-loop level, $Z^{\prime}$ can mix with photon via virtual $\ell^{+} \ell^{-}(\ell=\mu, \tau)$ pair production and annihilation diagrams. Through this mixing the dark matter can scatter off nucleons. To estimate the elastic scattering cross section for direct detection it is convenient to introduce effective operator [13]

$$
\mathscr{L}_{\text {eff }}=\frac{1}{\Lambda^{2}}\left(\bar{N}_{1} \gamma^{\mu} \gamma_{5} N_{1}\right)\left(\bar{\ell} \gamma_{\mu} \ell\right)
$$


where $\ell=\mu, \tau$. The cut-off scale $\Lambda$ is approximately given by $\Lambda=m_{Z^{\prime}} / g_{Z^{\prime}}$. As can be seen in the right panel of Fig. 3, the cut-off scale is in the electroweak scale. Due to Majorana nature of $N_{1}$, the vector current $\bar{N}_{1} \gamma^{\mu} N_{1}$ vanishes identically. The elastic scattering, however, is $p$-wave and the cross section is suppressed by $v_{\text {rel }}^{2} \approx 10^{-6}[13]$.

If we did not consider the muon $(g-2)$, the $U(1)_{\mu-\tau}$ gauge boson is also viable in the heavier $m_{Z^{\prime}}$ or larger $g_{Z^{\prime}}$ parameter region. In this case the $Z^{\prime}$ can be searched for at colliders through $4 \mu$, $2 \mu 2 \tau, 4 \tau$ production processes or missing $E_{T}$ signals in association with $2 \mu$ or $2 \tau$ events [11]. The parameter region with $m_{Z^{\prime}} \sim \mathscr{O}(10) \mathrm{GeV}$ and $g_{Z^{\prime}} \gtrsim 0.1$ is already sensitive $[14,5]$ to LHC searches, $Z \rightarrow 4 \mu[15,16]$. In the on-going LHC Run II experiment wider region of parameter space will be covered [17]. The region of our interest, i.e., $g_{Z^{\prime}} \sim \mathscr{O}\left(10^{-4}\right)$ and $m_{Z^{\prime}} \sim \mathscr{O}(100) \mathrm{MeV}$, may be searched for with dedicated study of specific topology of events including the one such as lepton jet [5]. This low $m_{Z^{\prime}}$ would be tested better at future high luminosity colliders such as FCC at CERN, Belle II, or planned neutrino facility LBNE.

The large $v_{\mu}$ flux from the dark matter annihilation at the galactic center can also be a signal of our model [11]. Those neutrinos can give additional contributions to the upward-going muon signals at the Super-Kamiokande. Although the photons emitted from the muons could contribute to the gamma rays from the galactic center, the cross section turns out to be too small to explain the possible excess of gamma ray events from the Fermi-LAT [18].

\section{Conclusions}

In this talk we considered dark matter phenomenology of right-handed neutrino dark matter candidate in an extension of Ma's scotogenic model with $U(1)_{\mu-\tau}$ gauge symmetry. We showed that we can explain the correct relic density of dark matter and the anomaly of muon $(g-2)$ at the same time. We need light $Z^{\prime}\left(m_{Z^{\prime}} \lesssim 400 \mathrm{MeV}\right)$ and small $U(1)_{\mu-\tau}$ gauge coupling $\left(3 \times 10^{-4} \lesssim g_{Z^{\prime}} \lesssim 10^{-3}\right)$. Although the gauge coupling constant is small we showed that the longitudinal polarization of $Z^{\prime}$ gauge boson in $N_{1} N_{1} \rightarrow Z^{\prime} Z^{\prime}$ annihilation process can give large enhancement factor $M_{1}^{4} / m_{Z^{\prime}}^{4}$ to get the correct relic abundance of dark matter. Our model is not strongly constrained by the direct detection experiments of dark matter. However, the $Z^{\prime}$ gauge boson can be searched for at the current LHC Run II and future high luminosity hadron or neutrino collider experiments.

\section{Acknowledgments}

This work is supported in part by National Research Foundation of Korea (NRF) Research Grant NRF-2015R1A2A1A05001869.

\section{References}

[1] M. Aoki, S. Kanemura and O. Seto, Phys. Rev. Lett. 102 (2009) 051805 [arXiv:0807.0361 [hep-ph]]; S. Kanemura and H. Sugiyama, Phys. Rev. D 86 (2012) 073006 [arXiv:1202.5231 [hep-ph]]; S. Baek, P. Ko, H. Okada and E. Senaha, JHEP 1409 (2014) 153 [arXiv:1209.1685 [hep-ph]]; M. Gustafsson, J. M. No and M. A. Rivera, Phys. Rev. Lett. 110 (2013) 21, 211802 [Phys. Rev. Lett. 112 (2014) 25, 259902] [arXiv:1212.4806 [hep-ph]]; M. Aoki, J. Kubo and H. Takano, Phys. Rev. D 87 (2013) 11, 
116001 [arXiv:1302.3936 [hep-ph]]; Y. Kajiyama, H. Okada and K. Yagyu, Nucl. Phys. B 874 (2013) 198 [arXiv:1303.3463 [hep-ph]]; S. Baek, H. Okada and T. Toma, JCAP 1406 (2014) 027 [arXiv:1312.3761 [hep-ph]]; S. Baek, H. Okada and T. Toma, Phys. Lett. B 732 (2014) 85 [arXiv:1401.6921 [hep-ph]]; S. Baek and H. Okada, arXiv:1403.1710 [hep-ph]; H. Okada, T. Toma and K. Yagyu, Phys. Rev. D 90 (2014) 095005 [arXiv:1408.0961 [hep-ph]]; S. Baek, arXiv:1410.1992 [hep-ph]; C. Q. Geng, D. Huang and L. H. Tsai, Phys. Rev. D 90 (2014) 11, 113005 [arXiv:1410.7606 [hep-ph]]; S. Baek and Z. F. Kang, arXiv:1510.00100 [hep-ph].

[2] S. Baek, H. Okada and K. Yagyu, JHEP 1504 (2015) 049 [arXiv:1501.01530 [hep-ph]].

[3] E. Ma, Phys. Rev. D 73 (2006) 077301 [hep-ph/0601225].

[4] S. Baek, Phys. Lett. B 756 (2016) 1 doi:10.1016/j.physletb.2016.02.062 [arXiv:1510.02168 [hep-ph]].

[5] W. Altmannshofer, S. Gori, M. Pospelov and I. Yavin, Phys. Rev. Lett. 113 (2014) 091801 [arXiv:1406.2332 [hep-ph]].

[6] S. Baek, P. Ko and W. I. Park, JHEP 1202 (2012) 047 [arXiv:1112.1847 [hep-ph]]; S. Baek, P. Ko, W. I. Park and E. Senaha, JHEP 1305 (2013) 036 [arXiv:1212.2131 [hep-ph]].

[7] G. W. Bennett et al. [Muon g-2 Collaboration], Phys. Rev. Lett. 92 (2004) 161802 [hep-ex/0401008].

[8] J. P. Miller, E. de Rafael and B. L. Roberts, Rept. Prog. Phys. 70 (2007) 795 [hep-ph/0703049].

[9] R. Foot, X. G. He, H. Lew and R. R. Volkas, Phys. Rev. D 50 (1994) 4571 [hep-ph/9401250].

[10] S. Baek, N. G. Deshpande, X. G. He and P. Ko, Phys. Rev. D 64 (2001) 055006 [hep-ph/0104141].

[11] S. Baek and P. Ko, JCAP 0910 (2009) 011 [arXiv:0811.1646 [hep-ph]].

[12] P. A. R. Ade et al. [Planck Collaboration], Astron. Astrophys. 571 (2014) A16 [arXiv:1303.5076 [astro-ph.CO]].

[13] N. F. Bell, Y. Cai, R. K. Leane and A. D. Medina, Phys. Rev. D 90 (2014) 3, 035027 [arXiv:1407.3001 [hep-ph]].

[14] K. Harigaya, T. Igari, M. M. Nojiri, M. Takeuchi and K. Tobe, JHEP 1403 (2014) 105 [arXiv:1311.0870 [hep-ph]].

[15] S. Chatrchyan et al. [CMS Collaboration], JHEP 1212 (2012) 034 [arXiv:1210.3844 [hep-ex]].

[16] G. Aad et al. [ATLAS Collaboration], Phys. Rev. Lett. 112 (2014) 23, 231806 [arXiv:1403.5657 [hep-ex]].

[17] F. del Aguila, M. Chala, J. Santiago and Y. Yamamoto, JHEP 1503 (2015) 059 [arXiv:1411.7394 [hep-ph]].

[18] F. Calore, I. Cholis and C. Weniger, JCAP 1503 (2015) 038 [arXiv:1409.0042 [astro-ph.CO]] and references therein. 